\title{
Microbiology of Periprosthetic Hip and Knee Infections in Surgically Revised Cases from 34 Centers in Mainland China
}

Hui-Ming Peng,' Zong-Ke Zhou, ${ }^{2}$ Fei Wang, ${ }^{3}$ Shi-Gui Yan, ${ }^{4}$ Peng $\mathrm{Xu},{ }^{5} \mathrm{Xi-Fu}$ Shang, ${ }^{6}$ Jia Zheng, ${ }^{7}$ Qing-Sheng Zhu, ${ }^{8}$ Li Cao,' Xi-Sheng Weng'

'Department of Orthopedics, Peking Union Medical College Hospital, CAMS \& PUMC, Beijing, 100730, People's Republic of China; ${ }^{2}$ Department of Orthopedic Surgery, West China Hospital, Sichuan University,

Chengdu, 61004I, Sichuan Province, People's Republic of China; ${ }^{3}$ Department of Orthopedic Surgery, The Third Hospital of Hebei Medical University, Shijiazhuang, 05005I, Hebei Province, People's Republic of China; ${ }^{4}$ Department of Orthopedic Surgery, Second Affiliated Hospital of Zhejiang University School of Medicine, Hangzhou, 310058 , Zhejiang Province, People's Republic of China; ${ }^{5}$ Department of Adult Joint Reconstruction, Xi' an Honghui Hospital, Xi' An, 7 I0054, Shaanxi Province, People's Republic of China; ${ }^{6}$ Department of Orthopedic Surgery, First Affiliated Hospital of University of Science and Technology of China, Hefei, 23000I, Anhui Province, People's Republic of China; ${ }^{7}$ Department of Orthopedic Surgery, Henan Provincial People's Hospital, Zhengzhou, 450003, Henan Province, People's Republic of China; ${ }^{8}$ Department of Orthopedic Surgery, Xijing Hospital of Air Force Medical University, Xi' An, 710032, Shanxi Province, People's Republic of China; ${ }^{\circ}$ Department of Orthopedic Surgery, The First Affiliated Hospital of Xinjiang Medical University, Urumqi, 830054, Xinjiang, People's Republic of China

Correspondence: Xi-Sheng Weng Department of Orthopedics, Peking Union Medical College Hospital, CAMS \&

PUMC, No. I Shuaifuyuan, Wangfujing,

Dongcheng District, Beijing, 100730,

People's Republic of China

Tel/Fax +86-10-69152710

Email drweng_wxs|377@I63.com
Background and Aim: Periprosthetic joint infection (PJI) is one of the most devastating complications after total joint arthroplasty (TJA). However, the antibiotic resistance of infecting pathogens can significantly vary in different parts of the country. In the current study, we analyzed the demographic and microbiological profiles of knee and hip PJI over three years and compared the microbiological differences between them.

Methods: A multicenter retrospective study of PJI patients in 34 referral medical centers in mainland China from January 2015 to November 2017 was performed.

Results: A total of 925 PJI patients were recruited, 452 were identified as knee PJIs, and 473 were hip PJIs. The most common causative pathogens were Staphylococcus aureus (26.5\%) and coagulase-negative staphylococci (14.3\%). Methicillin-resistant staphylococci were involved in 25.6\% (237/925) of all PJI cases. Mycobacterium and fungus only accounted for $6.5 \%(61)$ of all cases. Enteric gram-negative bacilli, anaerobes, and polymicrobial pathogens were more common in hip joint prostheses than in knee PJI $(P=0.014 ; P=$ $0.006 ; P=0.002$, respectively).

Conclusion: While the majority of causative pathogens in PJI cases are staphylococcal species, the prevalence of atypical organisms and resistant pathogens should also be given attention and warrant the need for empiric antibiotic treatment.

Keywords: arthroplasty, hips, knees, microorganism, prosthetic joint infection

\section{Introduction}

Prosthetic joint infection (PJI), while rare, is probably the most feared complication arising from lower extremity total joint arthroplasty (TJA), as it presents a significant therapeutic challenge and places a substantial socioeconomic burden on the health care system. ${ }^{1-3}$ The incidence of PJI after knee or hip replacement is approximately $1-2 \%, 4,5$ and the number of primary and revision TJA cases rapidly increases in aged people. Thus, the incidence of PJI is also expected to grow in the coming years. ${ }^{6}$

The use of appropriate antibiotics is the key to the successful treatment of PJI. Microbiological and epidemiological data can guide prophylactic and therapeutic antibiotic regimens, which can help reduce the risk of bacterial resistance to antibiotics. ${ }^{7}$ Different causative pathogens of PJI can lead to different treatment outcomes. For instance, it has been reported that methicillin-resistant Staphylococcus aureus (MRSA) PJI has a poorer prognosis and a higher risk of treatment failure. ${ }^{8}$ Furthermore, polymicrobial infections are associated with 
a higher risk of treatment failure, amputations, and infection-related mortality than infections caused by a single organism. ${ }^{9}$ Many studies have found significant differences in the microorganismal epidemiology of PJI in different centers. ${ }^{10,11}$ There are also notable microbiological differences depending on the location of the affected joint. ${ }^{1,7}$

An accurate understanding of the microbiology of postTJA PJIs is required to identify the most appropriate empiric antibiotics and therapeutic strategies. However, microbiological data and a comparison of different microorganisms involved in knee and hip PJIs are limited, especially in mainland China. This study, therefore, aims to compare the microbiological profiles between knee and hip PJIs in mainland China.

\section{Methods}

\section{Patients}

This retrospective cohort study included patients who received surgical treatment for knee and hip PJIs in 34 referral medical centers in mainland China between January 2015 and November 2017. This study was approved by the Institutional Review Board of Peking Union Medical College Hospital (S-K993). The study was conducted in compliance with the Declaration of Helsinki. Data after desensitization (hide patient name and contact information) then were entered into a computerized data management system. Only the chief PI and local PI got the access to all data in the system.

The criteria for eligible hospitals were: 1) referral hospitals where arthroplasty is performed in high volumes routinely (>500 hip/knee arthroplasty cases per year); 2) hospitals with enough technical and professional capability; 3) hospitals that can provide local Institutional Review Board (IRB) approval. At least one hospital was enrolled in each province of the Chinese mainland. A total of 41 research hospitals were selected, accounting for 3\% (41/ 1308) of the total number of Grade A tertiary hospitals in mainland China. To ensure the accuracy of the information, each research team appointed special personnel to check the data and contacted the investigation hospital to see if there were any empty entries or logical errors. All cases were followed for at least one year. Finally, 34 hospitals were enrolled in this retrospective study.

After the approval by the institutional review board, investigators searched the electronic databases in the hospital. They retrospectively reviewed patients' data with the
"International Classification of Diseases, Ninth Revision, Clinical Modification" code. The ICD-9-CM procedure codes $81.53,00.70,00.71,00.72,00.73$ were used to identify RTHAs, whereas the codes 81.55 , 00.80, 00.81 , $00.82,00.83,00.84$ were used to identify RTKAs associated with a principal diagnosis of PJI based on ICD9-CM diagnosis code 996.66. Two independent investigators reviewed the extracted data. Patients with primary septic arthritis or infection after internal fixation and open reduction were excluded. Due to the retrospective nature of the study, informed consent was waived.

\section{Definition of PJI}

PJI was defined according to the following criteria, which was modified from the international diagnostic standards (MSIS2013 or ICM2018): (1) isolation in one intraoperative culture of microorganisms, with evidence of infection at the site of the knee or prosthesis (presence of purulence in an affected joint, high synovial neutrophil percentage and synovial white blood cell count, elevated serum $\mathrm{C}$-reactive protein levels and serum erythrocyte sedimentation rate, or positive histological examination). (2) Isolation of the same microorganism from two or more cultures obtained from an infected prosthetic joint; (3) a sinus tract communicating with the prosthesis. PJI can be classified as early-onset (occurring within three months after surgery), delayed onset (occurring three months to two years after surgery), or late-onset (occurring two years after surgery) based on the time to infection. ${ }^{12}$ Polymicrobial PJI was defined as more than one microorganism isolated from one or more cultures of synovial fluid or periprosthetic tissues.

\section{Data Collection and Analysis}

The demographic characteristics, causative pathogens, time of infection, site of arthroplasty, and comorbidities were recorded. Where there was more than one infection episode on the same joint, only the first episode was recorded. The microbiological profiles of all infection cases were analyzed, and the difference in the proportion of causative pathogens between knee PJIs and hip PJIs was compared.

\section{Statistical Analysis}

All statistical analyses were two-sided and performed using the SPSS version 23 (IBM). Categorical data were 
analyzed by Fisher's exact test. A $P$-value of $<0.05$ was considered statistically significant.

\section{Results}

\section{Demographical Characteristics}

A total of 925 first-time infection cases (452 knee PJIs and 473 hip PJIs) from January 2015 to November 2017 were included in the retrospective study. There were 207 male patients with knee PJI (46\%) and 108 male patients with hip PJI (70\%). The hip PJI group had a mean age of 60.8 years and a male-to-female ratio of 2.1:1, whereas the knee PJI group had a male-to-female ratio of $0.84: 1$ and a mean age of 68.6 years. Bilateral joint infection was experienced by 14 patients with knee PJIs and eight patients with hip PJIs.

The incidence of alcoholism in patients with hip PJI was higher than in the knee PJI group (24\% vs $5 \% ; P=$ $0.01)$. However, the incidence of hypertension in patients with knee PJI was higher than in hip PJIs (55\% vs 33\%; $P<0.001)$. No difference in smoking, diabetes mellitus, cirrhosis, or malignancy was observed between the two groups. Also, there was no difference in the development of early-onset infection between patients with hip PJIs and those with knee PJIs $(22.6 \%$ vs $27.9 \% ; P=0.102)$ (Table 1).

\section{Microbiology}

The most common causative pathogens were $S$. aureus (26.5\%) and coagulase-negative staphylococci (14.3\%) (Table 2). Staphylococci had a high resistance rate to oxacillin or methicillin (Table 3). Methicillin-resistant coagulase-negative staphylococci were isolated in 98 patients (10.9\%), and MRSA was isolated in 97 cases (10.5\%). Up to $76 \%$ of coagulase-negative staphylococci (CoNS) and $40 \%$ of $S$. aureus were methicillin-resistant. Methicillinresistant staphylococci (MRS) was involved in $25.6 \%$ of all PJI cases.

It was found that the distribution of causative microorganisms in knee PJIs was 52\% aerobic gram positive, $7.3 \%$ gram negative, $2.2 \%$ fungus, $3.7 \%$ mycobacterium, $5.5 \%$ polymicrobial, and $29.0 \%$ negative culture. In hip PJIs, the distribution was $43.5 \%$ aerobic gram positive, $6.3 \%$ anaerobic, $8.8 \%$ gram negative, $2.5 \%$ fungus, $13.8 \%$ polymicrobial, and $24.9 \%$ negative culture. The proportion of causative microorganisms (anaerobic pathogens and polymicrobial pathogens) in knee PJIs was lower than that in hip PJIs (anaerobic pathogens: $P=0.002$;
Table I Demographics of Hip and Knee Prosthetic Joint Infections

\begin{tabular}{|l|c|c|c|c|c|}
\hline $\begin{array}{l}\text { Demographic } \\
\text { Characteristics }\end{array}$ & $\begin{array}{c}\text { Hip } \\
(\mathbf{n = 4 7 3 )}\end{array}$ & $\%$ & $\begin{array}{c}\text { Knee } \\
(\mathbf{n = 4 5 2})\end{array}$ & $\%$ & $\boldsymbol{P}$ \\
\hline Sex & 320 & 70 & 207 & 46 & $<0.001$ \\
Male & 153 & 30 & 245 & 54 & $<0.001$ \\
$\quad$ Female & $60.8 \pm 15.0$ & & $68.6 \pm 11.9$ & & $<0.001^{\mathrm{a}}$ \\
\hline Age (years), mean & $(21-82)$ & & $(32-80)$ & & \\
\pm SD (range) & & & & & \\
\hline Comorbidities & 156 & 33 & 248 & 55 & $<0.001^{\mathrm{a}}$ \\
Hypertension & 127 & 27 & 85 & 19 & 0.08 \\
Smoking & 89 & 19 & 117 & 26 & 0.26 \\
Diabetes mellitus & 113 & 24 & 22 & 5 & $0.01^{\mathrm{a}}$ \\
Alcoholism & 17 & 4 & 18 & 4 & 0.09 \\
Cirrhosis & 14 & 3 & 19 & 4 & 0.22 \\
Malignancy & & & & & \\
\hline Time to infection & 107 & 22.6 & 126 & 27.9 & 0.102 \\
Early onset & 170 & 35.9 & 150 & 33.2 & 0.939 \\
Delayed onset & 196 & 41.5 & 176 & 38.9 & 0.113 \\
Late onset & & & & & \\
\hline ASA grade & 62 & 13.1 & 36 & 7.8 & 0.022 \\
I & 90 & 19 & 100 & 22. & 0.0127 \\
\hline 2 & & 316 & 69.9 \\
3 & & & & \\
\hline
\end{tabular}

Note: ${ }^{\mathrm{A}} \mathrm{A}$ p value of $<0.05$ was considered to be statistically significant. Abbreviation: ASA grade, The American Society of Anesthesiologists physical Sstatus classification system.

polymicrobial pathogens: $P=0.01$ ), whereas the ratio of mycobacterium in knee PJIs was higher than that in hip PJIs $(P=0.01)$. There was no significant difference in the distribution of fungus, gram-positive and gram-negative microorganisms, and negative culture between hip and knee PJIs.

In polymicrobial hip PJI cases, $77.2 \%$ had two causative microorganisms, $4.5 \%$ had three, $9.0 \%$ had four, and $9.0 \%$ had five. In polymicrobial knee PJI cases, $100 \%$ had two causative microorganisms. S. aureus was the most common microorganism in polymicrobial hip PJIs. The causative microorganisms in polymicrobial infections are shown in Table 4.

\section{Discussion}

To our knowledge, this study is the most recent and most extensive cohort study that reported the microbiological data of post-TJA PJI, comparing the differences between knee and hip PJIs in mainland China. ${ }^{13,14}$ The study found that staphylococcal species were the most common organisms causing PJI. MRS was prevalent in both knee and hip PJIs. The prevalence of PJI-causing organisms was found 
Table 2 Microorganisms in Hip and Knee Periprosthetic Joint Infection

\begin{tabular}{|c|c|c|c|c|c|c|c|}
\hline Pathogen & $\begin{array}{l}\text { Hip and Knee } \\
{[n=925, n(\%)]}\end{array}$ & $\begin{array}{c}\text { Positive } \\
\text { Cultures } \\
\text { Rate } \\
(n=676, \%)\end{array}$ & $\begin{array}{c}\text { Hip } \\
{[n=473, n(\%)]}\end{array}$ & $\begin{array}{l}\text { Positive } \\
\text { Cultures } \\
\text { Rate } \\
(n=355, \%)\end{array}$ & $\begin{array}{c}\text { Knee } \\
{[n=452, n(\%)]}\end{array}$ & $\begin{array}{l}\text { Positive } \\
\text { Cultures } \\
\text { Rate } \\
(n=321, \%)\end{array}$ & $\mathbf{P}$ \\
\hline Aerobic gram-positive & $44 \mid(47.6)$ & 65.2 & $206(43.5)$ & 58.0 & $235(52.0)$ & 73.2 & 0.116 \\
\hline Staphylococcus aureus & $245(26.5)$ & 36.4 & III (23.5) & 31.1 & $134(30.0)$ & 43.2 & 0.169 \\
\hline Coagulase-negative & $132(14.3)$ & 19.6 & $72(15.2)$ & 20.3 & $60(13.3)$ & 18.9 & 0.667 \\
\hline \multicolumn{8}{|l|}{ Staphylococci } \\
\hline Streptococcus species & $40(4.4)$ & 6.1 & $15(3.1)$ & 4.2 & $25(5.5)$ & 8.4 & 0.248 \\
\hline Enterococcus faecalis & $8(0.8)$ & 0.9 & $3(0.6)$ & 0.8 & $5(1.1)$ & 1.1 & 0.907 \\
\hline Listeria monocytogenes & $8(0.8)$ & 0.9 & $3(0.6)$ & 0.8 & $5(1.1)$ & 1.1 & 0.907 \\
\hline Unspecified & $8(0.8)$ & 0.9 & $0(0.0)$ & 0.0 & $8(1.8)$ & 2.1 & 0.124 \\
\hline Anaerobes & $32(3.5)$ & 5.1 & $31(6.3)$ & 9.2 & I $(0.002)$ & 0.0 & $0.002^{\mathrm{a}}$ \\
\hline Propionibacterium species & $16(1.7)$ & 2.3 & $15(3.1)$ & 4.2 & $\mathrm{I}(0.002)$ & 0.0 & $0.038^{\mathrm{a}}$ \\
\hline Peptostreptococcus species & $13(1.4)$ & 1.9 & $13(2.5)$ & 3.4 & $0(0.0)$ & 0.0 & 0.064 \\
\hline Prevotella species & $3(0.4)$ & 0.5 & $3(0.6)$ & 0.8 & $0(0.0)$ & 0.0 & 0.356 \\
\hline Gram negative & $75(8.2)$ & 11.2 & $42(8.8)$ & 11.8 & $33(7.3)$ & 10.5 & 0.663 \\
\hline Escherichia coli & $26(2.8)$ & 3.7 & $18(3.8)$ & 5.0 & $8(1.7)$ & 2.1 & 0.229 \\
\hline Pseudomonas aeruginosa & $16(1.7)$ & 2.3 & $2(0.4)$ & 0.8 & $14(3.0)$ & 4.2 & 0.123 \\
\hline Pseudomonas stutzeri & $3(0.3)$ & 0.5 & $0(0.0)$ & 0.0 & $3(0.7)$ & 1.1 & 0.277 \\
\hline Serratia marcens & $13(1.4)$ & 1.9 & $6(1.3)$ & 1.7 & $7(1.5)$ & 2.1 & 0.869 \\
\hline Klebsiella pneumoniae & $9(1.0)$ & 1.4 & $9(1.9)$ & 2.5 & $0(0.0)$ & 0.0 & 0.109 \\
\hline Enterobacter cloacae & $3(0.3)$ & 0.5 & $3(0.6)$ & 0.8 & $0(0.0)$ & 0.0 & 0.356 \\
\hline Salmonella enterica & $3(0.3)$ & 0.5 & $3(0.6)$ & 0.8 & $0(0.0)$ & 0.0 & 0.356 \\
\hline Morganella morganii & $3(0.3)$ & 0.5 & $0(0.0)$ & 0.0 & $3(0.7)$ & 1.1 & 0.277 \\
\hline Fungus & $21(2.3)$ & 3.3 & $12(2.5)$ & 3.4 & $9(2.2)$ & 3.2 & 0.869 \\
\hline Candida species & $15(1.7)$ & 2.3 & $9(1.9)$ & 2.5 & $7(1.5)$ & 2.1 & 0.789 \\
\hline Unspecified & $6(0.6)$ & 0.9 & $3(0.6)$ & 0.8 & $2(0.7)$ & 1.1 & 0.907 \\
\hline Mycobacterium & $16(1.7)$ & 2.3 & $\mathrm{I}(0.0)$ & 0.0 & $15(3.7)$ & 5.3 & $0.014^{a}$ \\
\hline Mycobacterium tuberculosis & $2(0.7)$ & 0.9 & $0(0.0)$ & 0.0 & $2(1.5)$ & 2.1 & 0.124 \\
\hline M. tuberculosis complex & $2(0.7)$ & 0.9 & $0(0.0)$ & 0.0 & $2(1.5)$ & 2.1 & 0.124 \\
\hline Mycobacterium fortuitum & $\mathrm{I}(0.3)$ & 0.5 & $0(0.0)$ & 0.0 & I (0.7) & 1.1 & 0.277 \\
\hline Polymicrobial & $90(9.8)$ & 13.1 & $65(13.8)$ & 18.5 & $25(5.5)$ & 6.3 & $0.006^{\mathrm{a}}$ \\
\hline Negative cultures & $249(26.9)$ & & $118(24.9)$ & & $|3|(29.0)$ & & 0.391 \\
\hline Total & $925(100.0)$ & & $473(100.0)$ & & $452(100.0)$ & & \\
\hline
\end{tabular}

Note: ${ }^{a} \mathrm{~A} p$ value of $<0.05$ was considered to be statistically significant.

to be different between infected hip and knee joints: enteric gram-negative bacilli, anaerobes, and polymicrobial pathogens were more likely to occur in hip prosthetic joints than in knee cases.

Due to the difference in population baseline data and operation conditions in mainland China and abroad, the value of PJI microbiological epidemiology results reported in foreign literature is limited in China. Most of the previous microbiological data of PJI cases reported in mainland China were from a single center ${ }^{15-17}$ or studies with bias factors or a relatively small sample size. This caused a lack of recent PJI microbiological epidemic results in guiding the antibiotic treatment of PJI in mainland China. The present results will help improve this situation.

Several reports have shown that the microbiological profile may vary in different countries. In 2006, a retrospective case series analysis ${ }^{18}$ of 34 primary hip arthroplasties and 41 primary knee arthroplasties revised for PJI showed that staphylococci species were implicated in $61 \%$ of cases $(S$. aureus $=25 \%$, coagulase-negative Staphylococcus $=36 \%$ ). The mean time to diagnosis was 1.15 years. In 2001, it was found that the predominant infectious organisms were gram positive in 6489 knee replacements (Group B Streptococcus, Staphylococcus 
Table 3 Methicillin Resistance of Staphylococcal Periprosthetic Joint Infection

\begin{tabular}{|c|c|c|c|}
\hline Staphylococci & $\begin{array}{c}\text { Hip } \\
{[n=206, n(\%)]}\end{array}$ & $\begin{array}{c}\text { Knee } \\
{[n=235, n(\%)]}\end{array}$ & $P$ \\
\hline MSSA $^{a}$ & $72(35.0)$ & $107(45.5)$ & 0.71 \\
\hline MRSA $^{\mathrm{b}}$ & $51(24.8)$ & $68(28.9)$ & 0.75 \\
\hline MS-CoNS ${ }^{c}$ & $15(7.3)$ & $10(4.3)$ & 0.47 \\
\hline MR-CoNS ${ }^{d}$ & $68(33.0)$ & $50(21.3)$ & 0.12 \\
\hline Total & $206(100.0)$ & $235(100.0)$ & \\
\hline
\end{tabular}

Notes: a Methicillin-sensitive Staphylococcus aureus. '⿳亠口冋ethicillin-resistant S. aureus. ${ }^{c}$ Methicillin-sensitive coagulase-negative staphylococci. ${ }^{d}$ Methicillin-resistant coagulase-negative staphylococci.

Abbreviations: MSSA, methicillin-sensitive Staphylococcus aureus; MRSA, methicillin-resistant Staphylococcus aureus; MS-CoNS, methicillin-sensitive coagulasenegative staphylococci; MR-CoNS, methicillin-resistant coagulase-negative staphylococci.

epidermidis, and S. aureus). ${ }^{19}$ In 2009, a large European registry study investigated 426 infected knee arthroplasties recorded in the Swedish Knee Arthroplasty Register between 1986 and $2000 .{ }^{10}$ CoNS and S. aureus were the most common organisms $(27.5 \%$ and $30.5 \%$ of all cases, respectively). Staphylococci were involved in around 58\% of PJI cases, which was lower than what we reported in the present study. They also found a significant increase in staphylococcal resistance to methicillin over the duration.

In 2014, Aggarwal et al ${ }^{11}$ performed a retrospective study of deep PJI in two high-volume infection referral centers in the US and Europe. They studied intraarticular tissue cultures taken at the revision of primary knee and hip arthroplasty for indications of PJI. The study enrolled 921 patients with hip replacements and 749 patients with knee replacements. There was a significant difference in microorganism epidemiology between the two centers: the incidence of $S$. aureus infection was significantly higher in the US center $(31.0 \%)$ than in the European center (13.0\%). CoNS infections, however, were seen particularly more frequently in the European center $(39.3 \%)$ than in the US center $(20.2 \%)$. More recently (2016), Holleyman et $\mathrm{al}^{20}$ found that Staphylococcus was the most common organism isolated after the revision of a primary implant for infection (in $72 \%$ of all cases).

Certain differences in the proportions and types of pathogens have been observed with regard to the joint that is infected. The Mayo Clinic database shows that patients with hip arthroplasty have a lower frequency of $S$. aureus than coagulase-negative staphylococcal infection compared with those with infected knees, where the two types of staphylococci are relatively equal. ${ }^{7}$ Moreover, a previous study comprising 159 hip PJI and 135 knee PJI patients showed that anaerobic bacteria, enteric gram- negative bacilli, and polymicrobial pathogens are more frequently identified in hip than in knee infections, ${ }^{14}$ which was consistent with our findings. The possible reasons that may lead to the differences remain unknown. Further investigations are needed to explore the pathological mechanisms of pathogen infection at different joint types.

In the present study, methicillin-sensitive S. aureus (MSSA) and MS-CoNS were found to be potent pathogens and common causes of PJI. The antibiotic standard of care therapy for MSSA infections includes penicillinaseresistant penicillin (flucloxacillin/oxacillin/nafcillin), with first-generation cephalosporin and cefazolin as an alternative. $^{21,22}$ The third- or fourth-generation cephalosporins or cephalosporin may be used for MSSA infections for penicillin-allergic patients. ${ }^{23}$

However, several reports have shown an increase in the prevalence of resistant organisms. ${ }^{5,24}$ The present study showed that the occurrence of MRS was high, with $76 \%$ of CoNS and $40 \%$ of $S$. aureus being methicillin-resistant. In 2005, a retrospective case series analysis from Hong Kong ${ }^{16}$ characterized bacteria isolates between 1995 and 2003. The authors found no multiple-drug resistant isolates between 1995 and 1996; however, they observed a change in later years of the study. McLawhorn et $\mathrm{al}^{25}$ found that MRSA and MRSE combined accounted for $18.1 \%$ of PJI pathogens in the US Aggarwal et $\mathrm{al}^{11}$ showed that methicillin resistance was significantly more prevalent in the US than in Europe. These differences indicate geographical variability of organism prevalence, further addressing the need for registry data analysis at the population level. Owing to the high incidence of MRS in both infected hip and knee periprosthetic joints, physicians may need to select empiric antibiotics with broader coverage while waiting for culture results.

The incidence rates of polymicrobial infections in hip and knee prostheses were significantly different (65 vs 25 cases, $P=0.006$ ), which is in accordance with previous studies. ${ }^{9,14}$ Varying incidences (6 to 37\%) of polymicrobial infection have been reported. ${ }^{11,26}$ The literature also consistently demonstrated that patients with polymicrobial infections had inferior treatment outcomes. Tan et $\mathrm{al}^{27}$ showed that patients with polymicrobial PJI had a higher failure rate $(50.5 \%)$, as well as a higher rate of arthrodesis (odds ratio $[\mathrm{OR}]=11.06$ ), amputation $(\mathrm{OR}=3.80)$, and mortality $(\mathrm{OR}=7.88)$ compared with those with monomicrobial PJI. A series 
Table 4 Causative Microorganisms in Cases of First-Episode Polymicrobial Hip and Knee Periprosthetic Joint Infection

\begin{tabular}{|c|c|c|c|c|c|c|c|}
\hline Pathogen & $\begin{array}{c}\text { Hip } \\
\text { (n =65) }\end{array}$ & $\begin{array}{l}\text { Isolates } \\
\text { Rate } \\
(n=161)\end{array}$ & $\begin{array}{l}\text { Polymicrobial } \\
\text { Infections } \\
\text { Rate }(n=65)\end{array}$ & $\begin{array}{c}\text { Knee } \\
(n=25)\end{array}$ & $\begin{array}{c}\text { Isolates } \\
\text { Rate } \\
(n=50)\end{array}$ & $\begin{array}{c}\text { Polymicrobial } \\
\text { Infections } \\
\text { Rate }(n=25)\end{array}$ & $P$ \\
\hline \multicolumn{8}{|l|}{ Aerobic gram-positive } \\
\hline Staphylococcus aureus & 35 & 21.8 & 53.8 & 14 & 28.0 & 56.0 & 0.81 \\
\hline Coagulase-negative staphylococci & 15 & 9.1 & 22.7 & 4 & 8.0 & 8.0 & 0.93 \\
\hline Streptococcus species & 9 & 5.5 & 13.6 & 8 & 16.0 & 0.0 & 0.18 \\
\hline Enterococcus faecalis & 9 & 5.5 & 13.6 & 4 & 8.0 & 0.0 & 0.70 \\
\hline Enterococcus faecium & 3 & 1.8 & 4.5 & 4 & 8.0 & 0.0 & 0.64 \\
\hline Corynebacterium species & 6 & 3.6 & 9.1 & 4 & 8.0 & 0.0 & 0.48 \\
\hline Unspecified & 0 & 0.0 & 0.0 & 0 & 0.0 & 0.0 & $0.03^{\mathrm{a}}$ \\
\hline \multicolumn{8}{|l|}{ Anaerobes } \\
\hline Peptostreptococcus species & 15 & 9.1 & 22.7 & 0 & 0.0 & 0.0 & 0.28 \\
\hline Clostridium species & 3 & 1.8 & 4.5 & 0 & 0.0 & 0.0 & 0.64 \\
\hline Bacteroides fragilis & 3 & 1.8 & 4.5 & 0 & 0.0 & 0.0 & 0.64 \\
\hline Prevotella species & 3 & 1.8 & 4.5 & 0 & 0.0 & 0.0 & 0.64 \\
\hline Fusobacterium nucleatum & 3 & 1.8 & 4.5 & 0 & 0.0 & 0.0 & 0.64 \\
\hline Veillonella species & 3 & 1.8 & 4.5 & 0 & 0.0 & 0.0 & 0.64 \\
\hline \multicolumn{8}{|l|}{ Gram-negative } \\
\hline E. coli & 9 & 5.5 & 13.8 & 0 & 0.0 & 0.0 & 0.41 \\
\hline K. pneumoniae & 12 & 7.3 & 18.4 & 0 & 0.0 & 0.0 & 0.34 \\
\hline Enterobacter species & 6 & 3.6 & 9.6 & 4 & 8.0 & 12.0 & 0.48 \\
\hline Proteus vulgaris & 3 & 1.8 & 4.6 & 0 & 0.0 & 0.0 & 0.64 \\
\hline Salmonella enterica & 3 & 1.8 & 4.6 & 0 & 0.0 & 0.0 & 0.64 \\
\hline Pseudomonas aeruginosa & 3 & 1.8 & 4.6 & 4 & 8.0 & 16.0 & 0.23 \\
\hline Acinetobacter baumannii & 3 & 1.8 & 4.6 & 0 & 0.0 & 0.0 & 0.64 \\
\hline Aeromonas hydrophila & 3 & 1.8 & 4.6 & 0 & 0.0 & 0.0 & 0.64 \\
\hline Moraxella species & 0 & 0.0 & 0.0 & 4 & 8.0 & 16.0 & $0.03^{\mathrm{a}}$ \\
\hline Unspecified & 2 & 1.8 & 4.6 & 0 & 0.0 & 0.0 & 0.64 \\
\hline \multicolumn{8}{|l|}{ Fungus } \\
\hline Candida species & 3 & 1.8 & 4.6 & 0 & 0.0 & 0.0 & 0.64 \\
\hline Aspergillus versicolor & 3 & 1.8 & 4.6 & 0 & 0.0 & 0.0 & 0.64 \\
\hline \multicolumn{8}{|l|}{ Mycobacterium } \\
\hline Mycobacterium chelonae & 0 & 0.0 & 0.0 & 0 & 0.0 & 0.0 & 0.00 \\
\hline Total isolates & 161 & 100.0 & & 50 & 100.0 & & \\
\hline
\end{tabular}

Note: ${ }^{a} \mathrm{~A} p$ value of $<0.05$ was considered to be statistically significant.

analysis of 77 polymicrobial PJIs also demonstrated that $^{28}$ the infection-free rate after two years was $67.6 \%$ for polymicrobial infections as compared with $87.5 \%$ for monomicrobial infections. Additionally, Marculescu et $\mathrm{al}^{29}$ reported that the two-year cumulative probability of success of monomicrobial PJIs was $72.8 \%$, compared with $63.8 \%$ for polymicrobial PJIs. These findings offer three possible explanations for the increased failure rate in polymicrobial PJIs: (1) association with a soft tissue defect or a sinus tract; (2) frequent presence of difficult-to-treat organisms; ${ }^{27,29}$ (3) increased comorbidities.
Moreover, the total negative culture rate was high $(26.9 \%)$ in this study. A possible explanation for this might be the administration of antibiotics before obtaining culture samples. Many of the referral patients had already received antibiotic treatments, and therefore the medicines might not be held for at least two weeks when culture samples were obtained. Another possible reason is that culture samples obtained at the time of surgery were limited: some cases, for example, only had one swab culture sample. A minimum of three specimens for culture is recommended to maintain the same level of certainty in detecting PJIs. $^{21,30,31}$ A prospective study ${ }^{32}$ examined 297 
revision TJA procedures using multiple detection methods, and it was recommended that five or six specimens be collected to diagnose an underlying infection accurately. At least three samples should yield the growth of the underlying microorganism for adequate diagnosis of infection. According to the current department protocol, we collected at least three tissue samples, aspirated the synovial fluid, and injected it into blood culture bottles, improving the positive culture rate.

Despite the overall results obtained in the study, it had some limitations. First, there is a collection bias due to the retrospective design. As PJI cases that did not undergo revision surgeries were not included, data of this group infected by low-virulence pathogens may be missing. Second, we only included the first episode of PJI. Finally, although the study was performed at 34 referral hospitals in mainland China, some patients received primary arthroplasty and partial treatment for PJI at other institutions, which may have also caused selection bias. Hence, it is necessary to establish a national joint arthroplasty registry.

\section{Conclusion}

This study found that the primary organisms causing postTJA PJI are from the Staphylococcus genus. The prevalence of atypical organisms and resistant pathogens may warrant the need for empiric antibiotic therapy with broader coverage. The negative culture rate was high, so the current protocol on cultures needs improvement to maintain the same level of certainty. Fungal and mycobacterial PJI cases were found to be rare. Further work regarding PJI organism profiles is required, as it may confer significant clinical implications.

\section{Acknowledgments}

The authors are particularly grateful to all the panelists for their participation. Doctor Wei Huang, M.D., Yi-He Hu, M. D., Wan-Chun Wang, M.D., Tang Liu, M.D., Jin-Cheng Wang, M.D., Jian-Lin Zuo, M.D., Shu-Qing Tao, M.D., QiRong Qian, M.D., Ying-Zhen Wang, M.D., Tao Li, M.D., Zhi-Qiang Zhang, M.D., Ya-Yi Xia, M.D., Yong-Qing Xu, M.D., Rui He, M.D., Wei-Hua Xu, M.D., Xiao-Bin Tian, M. D., Jun Xiao, M.D., Wen-Bo Wang, M.D., Liao-Bin Chen, M. D., Qun-Hua Jin, M.D., Hong Yuan, M.D., Qing Jiang, M.D., Wen-Ming Zhang, M.D., Wei-Ming Liao, M.D., Zhan-Jun Shi, M.D., Jian-Zhong Xu, M.D. and Jian-Ning Zhao, M.D.

\section{Funding}

The authors received financial support for the research from key projects of National Natural Science Foundation of China (NO. 81630064).

\section{Disclosure}

The authors declare that they have no competing interests.

\section{References}

1. Kapadia BH, Berg RA, Daley JA, Fritz J, Bhave A, Mont MA. Periprosthetic joint infection. Lancet. 2016;387:386-394. doi:10.1016/S0140-6736(14)61798-0

2. Boniello AJ, Lieber AM, Courtney PM. Are patients who undergo THA for infection at higher risk for 30-day complications? Clin Orthop Relat Res. 2019;477:1624-1631. doi:10.1097/ CORR.0000000000000760

3. Brochin RL, Phan K, Poeran J, Zubizarreta N, Galatz LM, Moucha CS. Trends in periprosthetic hip infection and associated costs: a population-based study assessing the impact of hospital factors using national data. J Arthroplasty. 2018;33:S233-S238. doi:10.1016/j.arth.2018.02.062

4. Zahar A, Kendoff DO, Klatte TO, Gehrke TA. Can good infection control be obtained in one-stage exchange of the infected TKA to a rotating hinge design? 10-year results. Clin Orthop Relat Res. 2016;474:81-87. doi:10.1007/s11999-015-4408-5

5. Beam E, Osmon D. Prosthetic joint infection update. Infect Dis Clin North Am. 2018;32:843-859. doi:10.1016/j.idc.2018.06.005

6. Springer BD, Cahue S, Etkin CD, Lewallen DG, McGrory BJ. Infection burden in total hip and knee arthroplasties: an international registry-based perspective. Arthroplast Today. 2017;3:137-140. doi:10.1016/j.artd.2017.05.003

7. Tande AJ, Patel R. Prosthetic joint infection. Clin Microbiol Rev. 2014;27:302-345. doi:10.1128/CMR.00111-13

8. Peng HM, Wang LC, Zhai JL, Weng XS, Feng B, Wang W. Effectiveness of preoperative decolonization with nasal povidone iodine in Chinese patients undergoing elective orthopedic surgery: a prospective cross-sectional study. Braz J Med Biol Res. 2017;51: e6736. doi:10.1590/1414-431 x20176736

9. Flurin L, Greenwood-Quaintance KE, Patel R. Microbiology of polymicrobial prosthetic joint infection. Diagn Microbiol Infect Dis. 2019;94:255-259. doi:10.1016/j.diagmicrobio.2019.01.006

10. Stefánsdóttir A, Johansson D, Knutson K, Lidgren L, Robertsson O. Microbiology of the infected knee arthroplasty: report from the Swedish Knee Arthroplasty Register on 426 surgically revised cases. Scand J Infect Dis. 2009;41:831-840. doi:10.3109/ 00365540903186207

11. Aggarwal VK, Bakhshi H, Ecker NU, Parvizi J, Gehrke T, Kendoff D. Organism profile in periprosthetic joint infection: pathogens differ at two arthroplasty infection referral centers in Europe and in the United States. J Knee Surg. 2014;27:399-406. doi:10.1055/s-0033-1364102

12. Peel TN, Cheng AC, Choong PF, Buising KL. Early onset prosthetic hip and knee joint infection: treatment and outcomes in Victoria, Australia. $J$ Hosp Infect. 2012;82:248-253. doi:10.1016/j. jhin.2012.09.005

13. Peng HM, Wang LC, Cheng JY, et al. Rates of periprosthetic infection and surgical revision in Beijing (China) between 2014 and 2016: a retrospective multicenter cross-sectional study. J Orthop Surg Res. 2019;14:463. doi:10.1186/s13018-019-1520-3

14. Tsai Y, Chang CH, Lin YC, Lee SH, Hsieh PH, Chang Y. Different microbiological profiles between hip and knee prosthetic joint infections. J Orthop Surg (Hong Kong). 2019;27. doi:10.1177/ 2309499019847768 
15. Peng HM, Weng XS, Zhai JL, et al. [The microbiology and antimicrobial susceptibility of the infected knee arthroplasty]. Zhonghua Wai Ke Za Zhi. 2013;51:413-416. Chinese.

16. Ip D, Yam SK, Chen CK. Implications of the changing pattern of bacterial infections following total joint replacements. J Orthop Surg (Hong Kong). 2005;13:125-130. doi:10.1177/230949900501300204

17. Tsai JC, Sheng WH, Lo WY, Jiang CC, Chang SC. Clinical characteristics, microbiology, and outcomes of prosthetic joint infection in Taiwan. $J$ Microbiol Immunol Infect. 2015;48:198-204. doi:10.1016/j.jmii.2013.08.007

18. Phillips JE, Crane TP, Noy M, Elliott TS, Grimer RJ. The incidence of deep prosthetic infections in a specialist orthopaedic hospital: a 15-year prospective survey. J Bone Joint Surg Br. 2006;88:943-948. doi:10.1302/0301-620X.88B7.17150

19. Peersman G, Laskin R, Davis J, Peterson M. Infection in total knee replacement: a retrospective review of 6489 total knee replacements. Clin Orthop Relat Res. 2001;392:15-23. doi:10.1097/00003086200111000-00003

20. Holleyman RJ, Baker P, Charlett A, Gould K, Deehan DJ. Microorganisms responsible for periprosthetic knee infections in England and Wales. Knee Surg Sports Traumatol Arthrosc. 2016;24:3080-3087. doi:10.1007/s00167-015-3539-2

21. Osmon DR, Berbari EF, Berendt AR, et al.; Infectious Diseases Society of America. Diagnosis and management of prosthetic joint infection: clinical practice guidelines by the Infectious Diseases Society of America. Clin Infect Dis. 2013;56:e1-e25. doi:10.1093/ $\mathrm{cid} / \mathrm{cis} 803$

22. Sharff KA, Graber CJ, Spindel SJ, Nguyen HM. Ceftriaxone for methicillin-sensitive Staphylococcus aureus osteoarticular infections: a survey of infectious disease physicians' attitudes and review of the literature. Infect Dis Clin Pract. 2014;22:132-140. doi:10.1097/ IPC.0000000000000109

23. Campagna JD, Bond MC, Schabelman E, Hayes BD. The use of cephalosporins in penicillin-allergic patients: a literature review. $J$ Emerg Med. 2012;42:612-620. doi:10.1016/j.jemermed.20 11.05 .035
24. Bradbury T, Fehring TK, Taunton M, et al. The fate of acute methicillin-resistant Staphylococcus aureus periprosthetic knee infections treated by open debridement and retention of components. J Arthroplasty. 2009;24:101-104. doi:10.1016/j.arth.2009.04.028

25. McLawhorn AS, Nawabi DH, Ranawat AS. Management of resistant, atypical and culture-negative periprosthetic joint infections after hip and knee arthroplasty. Open Orthop J. 2016;10:615-632. doi:10.2174/1874325001610010615

26. Tariq F, Segreti J. Microbiology of Periprosthetic Joint Infection. Periprosthetic Jt. Infect. Hip Knee. Vol. 9781461479284. Springer: New York; 2014: 97-105. doi:10.1007/978-1-4614-7928-4_8

27. Tan TL, Kheir MM, Tan DD, Parvizi J. Polymicrobial periprosthetic joint infections: outcome of treatment and identification of risk factors. J Bone Joint Surg Am. 2016;98:2082-2088. doi:10.2106/ JBJS. 15.01450

28. Wimmer MD, Friedrich MJ, Randau TM, et al. Polymicrobial infections reduce the cure rate in prosthetic joint infections: outcome analysis with two-stage exchange and follow-up $\geq$ two years. Int Orthop. 2016;40:1367-1373. doi:10.1007/s00264-015-2871-y

29. Marculescu CE, Cantey JR. Polymicrobial prosthetic joint infections: risk factors and outcome. Clin Orthop Relat Res. 2008;466:1397-1404. doi:10.1007/s11999-008-0230-7

30. Peel TN, Spelman T, Dylla BL, et al. Optimal periprosthetic tissue specimen number for diagnosis of prosthetic joint infection. $J$ Clin Microbiol. 2016;55:234-243. doi:10.1128/JCM.01914-16

31. Vasoo S, Munson E. Improving the diagnosis of orthopedic implant-associated infections: optimizing the use of tools already in the box. J Clin Microbiol. 2018;56:e01379-18. doi:10.1128/ JCM.01379-18

32. Atkins BL, Athanasou N, Deeks JJ, et al.; The OSIRIS Collaborative Study Group. Prospective evaluation of criteria for microbiological diagnosis of prosthetic-joint infection at revision arthroplasty. $J$ Clin Microbiol. 1998;36:2932-2939. doi:10.1128/JCM.36.10.29322939.1998
Infection and Drug Resistance

\section{Publish your work in this journal}

Infection and Drug Resistance is an international, peer-reviewed openaccess journal that focuses on the optimal treatment of infection (bacterial, fungal and viral) and the development and institution of preventive strategies to minimize the development and spread of resistance. The journal is specifically concerned with the epidemiology of

\section{Dovepress}

antibiotic resistance and the mechanisms of resistance development and diffusion in both hospitals and the community. The manuscript management system is completely online and includes a very quick and fair peerreview system, which is all easy to use. Visit http://www.dovepress.com/ testimonials.php to read real quotes from published authors. 